detect possible erratic behavior in the $H_{\alpha}$ region; results were negative. At the Flower and Cook Observatory, Fliegel continued two-color photoelectric observations of these stars.

Mrs. Bookmyer used the 28 -inch reflector to make photoelectric observations of SW Lac in two wavelength regions. Five light curves each, in yellow and blue light have been obtained. The shape of the light curve varies with time. There is a change in the sign of the $\cos \theta$ term.

Brown made a period study of RZ Dra using the observations of Dr. Chou. James Louden, Gary Lampert, and Robert Richardson began studies of RR Cen, $\delta$ Cap, and AL Vel, respectively, using photoelectric observations made earlier by Dr. Wood at the Mount Stromlo Observatory. The light curves are not complete and orbital solutions are not expected.

We wish to express our thanks to the many New Zealand individuals and organizations who have aided our work and to the U.S. Naval Observatory for loan of the telescopes. Grants from the Grants and Awards Committee of the Institute of Cooperative Research and the Research Committee, both of the University of Pennsylvania, have helped in various phases of our work. For aid, not only with the site survey, but in many other parts of our scientific programs we wish to acknowledge the help received from the National Science Foundation.

Frank Bradshaw Wood, Director

National Radio Astronomy Observatory, Green Bank, West Virginia

Dr. Otto Struve resigned as Director of the Observatory in December 1961. His scientific leadership was an inspiration to the entire staff during his two years at the NRAO, and his retirement is regretted by all.

Upon Dr. Struve's resignation, D. S. Heeschen was named Acting Director.

The NRAO Visiting Committee consisted of G. W. Swenson (University of Illinois, Chairman), J. W. Firor (High Altitude Observatory), H. Friedman (NRL), C. H. Mayer (NRL), D. E. Osterbrock (Washburn Observatory), C. PayneGaposchkin (Harvard), G. Stanley (Cal Tech), and B. Strömgren (Institute for Advanced Study).

PERSONNEL

C. R. Lynds and T. K. Menon resigned in December. T. Orhaug returned to Sweden in July, after a two-year stay at the Observatory as research associate.

D. E. Hogg accepted a position as assistant scientist, effective September 1961. H. M. Johnson accepted a position as associate scientist, effective
July 1962. During the course of the year D. J. Crampin, V. R. Venugopal, I. Pauliny-Toth, and B. Höglund arrived at the NRAO for varying stays as research associates.

R. Dugatkin and E. M. Filloy, electronics engineers from Argentina, came to the Observatory in September 1961 for a one-year visit. On their return to Argentina they will be associated with a new radio astronomy project being developed jointly by several groups in Argentina.

The engineering staff of the Observatory has been considerably strengthened during the year. J. L. Dolan (April), B. Hansson (July), and A. M. Shalloway (August) joined the staff as electronics engineers. W. G. Horne joined the staff as structural engineer in April. M. M. Small, on leave from Brookhaven National Laboratory, was named head of the $140-\mathrm{ft}$ project early in the year.

About 20 graduate and undergraduate students from a number of universities spent the summer at Green Bank in various capacities.

\section{RESEARCH PROGRAMS}

Planetary Observations. Drake continued his work on Venus, Saturn, and Jupiter. Carefully calibrated observations of Venus were made at $10-\mathrm{cm}$ wavelength during the period surrounding the 1961 inferior conjunction. These indicated that the radiation from Venus was nearly constant with time, showing no detectable short-term variations. Over the long term, there appeared to be a small variation in mean blackbody disk temperature $T_{\mathrm{BB}}$ with phase angle, such that $T_{\mathrm{BB}}$ could be expressed as $T_{\mathrm{BB}}=622+39 \cos \left(i \pm 17^{\circ}\right)^{\circ} \mathrm{K}$, when the $+\operatorname{sign}$ is taken for values of $i$ occurring after inferior conjunction, and the - sign for values occurring before. These results seem to support a greenhouse theory for the Venus atmosphere.

Extensive observations were made at $10 \mathrm{~cm}$ near the time of superior conjunction in 1962. These produced a mean value of $T_{\mathrm{BB}}=610^{\circ} \pm 55^{\circ} \mathrm{K}$, consistent with the results given above, and giving strong evidence that the phase effect at $10 \mathrm{~cm}$ is very small.

Saturn was observed at $10 \mathrm{~cm}$, and a mean value $T_{\mathrm{BB}}=196^{\circ} \mathrm{K}$, with a mean error of $56^{\circ} \mathrm{K}$, was found. This may be interpreted as being a result of a relatively high temperature deep in the Saturn atmosphere, or as a result of weak radiation from a radiation-belt system.

Jupiter was observed extensively in both the 1961 and 1962 apparitions at $10-\mathrm{cm}$ wavelength. No evidence for long-term variation could be found, and the change in the mean radio emission over one year appears to be less than $1 \%$. However, there is a systematic change in intensity, amounting to a peak-to-peak variation of about $10 \%$, with the 
longitude of central meridian. This variation is synchronized with the low-frequency radio-source system, and so correlated with System III longitudes. The observed variation in intensity with longitude is irregular, and has no obvious correlation other than in period with the decameter radio results, or the decimeter longitude dependent effects observed previously on lower frequencies. Studies of this phenomenon are in progress.

Galactic Studies. D. E. Hogg completed his analysis of the ionized hydrogen complex IC 1795, IC 1805, and IC 1848. This work was part of Hogg's $\mathrm{Ph} . \mathrm{D}$. thesis at the University of Toronto.

The entire region was surveyed with the $85-\mathrm{ft}$ telescope at $1400 \mathrm{Mc}$ (in the continuum) and at $1402 \mathrm{Mc}$ (in the hydrogen line). Additional observations of IC 1795 were made at 3000 and 8000 $\mathrm{Mc} / \mathrm{sec}$ and of IC 1805 at $3000 \mathrm{Mc} / \mathrm{sec}$. The calibrations of the systems used were supplied by the staff of the Observatory. The scale of absolute intensity depends ultimately on the flux of Cassiopeia A measured with the standard horn.

From the observed intensity contours the distribution of electron density within each nebula was derived, under the assumption that the electron temperature in the nebulae is $10^{4} \mathrm{~K}$. The models for IC 1795 and IC 1805 are quite detailed; because observations of IC 1848 were made at $1400 \mathrm{Mc} / \mathrm{sec}$ only, where the beamwidth of the telescope is $36^{\prime}$, the model for it is necessarily crude. The important features of these models are that a large fraction of the radio emission from IC 1805 originates in an ellipsoidal shell centered on the star cluster, and that IC 1795 is composed of two small regions of ionization, each of which is partially obscured by the absorbing matter which lies in front of the nebula. These models were checked by comparing, at several lower frequencies, the flux predicted by the model with that which is observed.

The amount of ultraviolet radiation beyond the Lyman limit radiated by the exciting stars has been compared with the amount required to support ionization within the nebula, as deduced from the radio observations. IC 1805 is quite clearly densitybounded, i.e., a significant fraction of the stellar radiation escapes, whereas IC 1848 is more nearly a classical Strömgren region. IC 1795 is an enigma, since its single O8 star cannot maintain the amount of ionized material required by the radio observations. However, there is at present no indication of a nonthermal component in the radiation from the nebula; it seems that there must be another $\mathrm{O}$ star which is hidden by the heavy absorption in the region.

The $1420-\mathrm{Mc} /$ sec observations of neutral hydrogen have been of limited value in the interpretation of the continuum observations. The problem is that since the $H$ II complex lies near the galactic plane, it is difficult to identify features in the line profile which are associated with the complex.

S. Weinreb, a graduate student from MIT, used the 85 - $\mathrm{ft}$ telescope and a novel new receiving system designed and built by him in attempts to measure the galactic deuterium to hydrogen ratio, and Zeeman splitting of the $21-\mathrm{cm}$ hydrogen line. The receiving system incorporates two techniques: (1) The autocorrelation function of the signal is determined digitally and Fourier transformed by a computer to give the power spectrum. Use of this method gives high stability and permits multichannel operation (the spectrum is measured at many frequencies during the same time interval); (2) The effective observation time is quadrupled by using four switched receivers to monitor both polarizations all of the time. Experimental determination of the sensitivity of this autocorrelation system showed that the rms fluctuation of the output was approximately 1.4 times the theoretical value, for integration times of the order of several weeks.

In the deuterium line observations, Weinreb observed Cas A with the $85-\mathrm{ft}$ telescope $12 \mathrm{~h}$ a day for nine weeks, in an attempt to detect the deuterium line in absorption in the spectrum of the source. From his negative results, he obtained an upper limit to the deuterium to hydrogen ratio of $1 / 13000$, a value significantly smaller than previously determined upper limits.

Weinreb's observations for Zeeman splitting of the $21-\mathrm{cm}$ line of hydrogen also gave negative results. He found that the line of sight component of the magnetic field must be less than $3 \times 10^{-6} \mathrm{G}$ in the direction of Cas $A$ and less than $5 \times 10^{-6} \mathrm{G}$ in the direction of $\mathrm{Tau} A$, results which are consistent with those obtained at other observatories.

Galaxies and Discrete Sources. A series of observations of possible supernovae remnants, begun in 1961 by C. R. Lynds, has been continued by D. E. Hogg. The program at $1400 \mathrm{Mc} / \mathrm{sec}$ has been completed, and is in progress at 750 and $3000 \mathrm{Mc} / \mathrm{sec}$.

Most of the observational effort has been devoted to the determination of the spectra of and radio brightness distribution across the four extended sources HB 21, HB 9, S 147, and CTA 1. The latter three are of special interest, since it has been suggested that they have positive spectral indices. Although the specification of the spectral indices must await the completion of the $3000-\mathrm{Mc} / \mathrm{sec}$ observations, the preliminary results at the other two frequencies suggest that the indices are in fact negative, for all three of the nebulae.

In the course of this program, six smaller objects which had been considered, on the basis of their optical appearance, as supernovae remnants, were also observed. Three of them, NGC 6888 and Sharpless Nos. 188 and 274, were not detected. The other three, detected both at 1400 and $750 \mathrm{Mc} / \mathrm{sec}$, 
are the following: van den Bergh No. 9, a nebula included in van den Bergh's list and which contributes part of the flux of the radio source CTB 1 ; Sharpless No. 224, also included in van den Bergh's list; and NGC 2359, mentioned as a possible remnant by Minkowski. Detection of NGC 2359 is of particular importance, since there is a Wolf-Rayet star, HD 56925, at its center. It has not yet been established, however, whether the spectrum of this source is thermal or nonthermal.

Heeschen completed his observations with the 85-ft telescope of normal galaxies, at 10-, 21-, 40-, and $68-\mathrm{cm}$ wavelengths. Radio emission was detected from 18 galaxies, while 19 yielded negative results. All of the galaxies detected were late-type spirals or irregular galaxies. The radio spectra of these galaxies are similar to the spectra of nonthermal discrete sources in general. A mean spectral index of -0.93 was found from the 14 galaxies for which reasonably consistent data are available at several wavelengths. The range in spectral index is small - only two spectra have indices which differ from the mean by more than \pm 0.3 . The mean absolute photographic magnitude of the galaxies observed is about -19 , with a total range of only \pm 1 mag. The dispersion in absolute radio magnitude, on the other hand, is considerably greater, with a total range of $\pm 2.5 \mathrm{mag}$.

Wade made a search for radio emission from galaxies in the Virgo cluster, using the 85 -ft telescope at $10-\mathrm{cm}$ wavelength. Forty-four of the brighter members of the cluster were examined. Radio emission was detected from seven galaxies which had not previously been detected. These are: NGC 4254, 4303, 4321, 4435, 4501, 4550, and 4569. Of these, all but two, NGC 4435 and NGC 4550 , are late-type spirals and have radio indices $m_{\mathrm{r}}-m_{\mathrm{pg}}$ of about 0.5. NGC 4435 is an SB0 and NGC 4550 is an E7 type galaxy. Both have radio indices of about -1.8 , and are probably not "normal" radio emitters.

Atmospheric Effects. T. Orhaug initiated an observing program to investigate the effects of clouds, rain, and water vapor content of the atmosphere on radio-astronomical observations at short wavelengths. Observations have been made almost daily since December 1961 at a wavelength of $3.75 \mathrm{~cm}$. Analysis of these observations shows that shortterm fluctuations in brightness temperature of the order of $1^{\circ} \mathrm{K}$ and more occur on an average for about $21 \%$ of total observing time. There is a strong correlation between the amplitude of the fluctuations and clouds. Fluctuations of greater than $1^{\circ} \mathrm{K}$ may also occur on clear days, however, presumably due to variations in water vapor content of the atmosphere.

This work is now being continued by V. R. Venugopal. Observations will be made at Green Bank at 6-cm wavelength, and then the experiment will be moved to a drier site and similar observations made for comparison purposes.

M. Vinokur is studying amplitude and phase scintillations at microwave frequencies, due to the earth's atmosphere, from the standpoint of their possible effect on very high resolution antenna systems.

Injormation Theory. M. Vinokur has investigated problems of sampling theory and information retrieval, as they relate to radio-astronomy observations in general, and in particular as they may affect the design of radiometer output systems. He has studied the question of the optimum rate of sampling of a signal mixed with noise, and developed a general method of analyzing quantitatively the combined effects of the filtering and sampling processes on the signal-to-noise ratio. His results have been applied to the design of output systems for multichannel hydrogen-line receivers. $\mathrm{He}$ is now studying, experimentally and by computer analysis, methods for the restoration of a signal in the presence of noise.

INSTRUMENTATION

140-Foot Telescope. The 140-ft telescope project underwent major contractual, management, and engineering changes during the course of the year. The contract with the original prime contractor was canceled by mutual agreement. Stone \& Webster Engineering Corporation has been engaged to provide over-all engineering management of the job, and M. M. Small has been appointed project manager for the Observatory and Associated Universities, Inc. A number of design changes have been made, to facilitate fabrication or erection, and to provide a stronger structure. Basically, however, the design remains the original one of N. L. Ashton, and the performance specifications of the telescope have not been altered. Contracts have been let for fabrication and erection of all major components, and work is proceeding on schedule in the various contractors' shops and at Green Bank.

300 -Foot Telescope. Work on the 300-ft telescope has progressed steadily, under the direction of J. W. Findlay. Erection of the steel superstructure was completed in early December 1961. During the winter and spring of 1962 the drive system was installed, the superstructure was measured, and the surface planned and designed. This latter work included radio-frequency measurements of various wire meshes, and computer analysis on fitting the parabolic surface to the superstructure. The surface was installed during the summer, and it is expected that the instrument will go into operation this fall.

Miscellaneous. The $40-\mathrm{ft}$ transit telescope was completed during the winter, and regular daily observations were begun in March. The telescope, 